\title{
Fabrication of the Microcontainers
}

A $5.5 \mu$ m-thick sacrificial layer of poly(methylmethacrylate) (PMMA, MW: 996K) [Sigma-Adlrich, www.sigma-aldrich.com] was spun on a silicon wafer. On top of the PMMA-coated wafer, a $15 \mathrm{~nm}$ adhesion-promoting chromium (Cr) layer and a $100 \mathrm{~nm}$ conductive seed copper $(\mathrm{Cu})$ layer were evaporated. After the thin film deposition, we spin coated a layer of Shipley SPR220 7.0 photoresist [Rohm and Haas, www.rohmhaas.com]. The thickness of the photoresist was controlled via the spin speed and by varying the number of coatings applied. After a soft bake, the resist was exposed to UV light using an Ultra $\mu$ line Series Quintel mask aligner [Quintel Corp., www.quintelcorp.com] and patterned using a transparency mask. After developing the photoresist, electrodeposition was used to grow the metallic frames of the microcontainers within the photoresist mold to a height of 6-15 $\mu \mathrm{m}$ (depending on the characteristics required by various applications). We used commercial electrolytic solutions that contained the metal ions of choice [Technic, Inc., www.technic.com] to electrodeposit different metals. For the construction of non-magnetic containers, $\mathrm{Cu}$ was electroplated, and for magnetic containers, Ni was used. A second round of photolithography was performed in order to pattern the hinges. A layer of SPR220 was spun on top of the substrate and exposed to the hinge mask. Wider, internal hinges were located between adjacent faces, whereas the thinner, external hinges resided at the outer edges of the frames. Alignment marks were used to ensure alignment of the hinges to the frames of the 2D precursors. After the hinge patterns were developed in 451 Developer, the exposed $\mathrm{Cu}$ (seed) and $\mathrm{Cr}$ (adhesion) regions in between the electrodeposited frames were etched using commercial etchants (APS-100 for $\mathrm{Cu}$ and CRE-473 for Cr [Technic, Inc., www.technic.com]). Tin/lead (60/40, m.p. 183 ${ }^{\circ} \mathrm{C}$ ) solder [Technic, Inc., www.technic.com] was then electroplated into the hinge regions. The height of the hinges was approximately $16 \mu \mathrm{m}$. After the solder electrodeposition, the photoresist layers were stripped off with acetone, the remaining $\mathrm{Cu}$ seed and $\mathrm{Cr}$ adhesion layers were etched and the 2Dprecursor template composed of metal frames connected with solder hinges was immersed in N-Methyl Pyrrolidone (NMP) [Sigma-Adlrich, www.sigma-aldrich.com] to dissolve the sacrificial PMMA layer and release the precursors from the wafer. Approximately 50 precursors in NMP were spread across a small crystallization dish and a small amount of \#5 RMA flux [Indium Corporation, www.indium.com] was added to dissolve any solder oxides that may have formed. The dish was heated to $100{ }^{\circ} \mathrm{C}$ for 3 minutes and then ramped up to $250{ }^{\circ} \mathrm{C}$ for approximately 90 seconds until the solder became molten. During reflow, if the solder wet the top layer of metal on the 2D precursor, the fabrication yields were poor. Solder wet copper well but did not we $\mathrm{Ni}$ well, hence for containers with $\mathrm{Cu}$ frames it was necessary to add a thin Ni layer to improve yields. When solder reflowed, the molten solder at the hinges and generated the torque to fold the 2D precursors into 3D microcontainers. Upon cooling, the solder solidified and permanently held the container frames together.

\section{Container Loading}

We used two methods to load reagents into the containers, depending on the wettability of the chemical reagent on the container. When the chemical wet the container well, we simultaneous loaded several boxes by immersing them in a drop of the chemical reagent. The solvent was removed by evaporation. This left behind the polymer [Pluronic ${ }^{\circledR}$ F68, BASF, www.basf.com] soaked with the chemical reagent.

The second method utilized two three-axes Newport micromanipulators [Models 460A \& M462, www.newport.com] to independently control the position of the microcontainer and the syringe [World Precision Instruments, Inc. Nanofil ${ }^{\mathrm{TM}}$ Syringe, www.wpiinc.com]. The syringe was outfitted with a 36gauge needle [WPII 36 Gauge Needle, www.wpiinc.com] to facilitate loading of the microcontainers.

\section{Chemical Release \& Reaction Specifics}

Red Dye Diffusion Experiment (Fig. 2a-b): We loaded the containers with a mixture composed of: $1.6 \mathrm{~mL}$ (0.261g) FD\&C Red 40 [McCormick \& Co., Inc., www.mccormick.com] and an aqueous polymeric solution composed of $1.0 \mathrm{~g}$ of Pluronic F68 dissolved in $10 \mathrm{~mL}$ of water (18.4 M $\Omega$ ) . A 2:1:2 (by volume) mixture of glycerol:ethanol:water was used as the diffusion medium and this medium was added to a 
small chamber containing the loaded microcontainer. The diffusion profiles were imaged using a stereozoom binocular microscope.

Magnetically-Guided Phenolphthalein-KOH Reaction (Fig. 2c): The indicator mixture for the phenolphthalein-KOH reaction was prepared by adding $0.25 \mathrm{~mL}$ of phenolphthalein solution $(0.5 \mathrm{~g}$ of phenolphthalein [Frey Scientific, www.freyscientific.com] in $100 \mathrm{~mL}$ of $95 \%$ ethanol) to an aqueous polymeric solution composed of $1.0 \mathrm{~g}$ of Pluronic F68 dissolved in $10 \mathrm{~mL}$ water and loaded into a nickelbased microcontainer. The microcontainer was placed into a well of a tissue culture plate [Falcon ${ }^{\circledR}$ Multiwell $^{\mathrm{TM}}$ Tissue Culture Plate, 24 Well, www.bdbiosciences.com], and a 1:1:1 (by volume) glycerol: water: $1 \mathrm{M} \mathrm{KOH}(\mathrm{aq})$ medium was introduced into the chamber. The microcontainer was guided and controlled using a 0.35 pull lb., 1/8” diameter AlNiCo round bar magnet [McMaster-Carr, www.mcmaster.com].

\section{Copper (II) Sulfate Pentahydrate-KOH Reaction (Fig. 3a-c):}

$\mathrm{CuSO}_{4}(\mathrm{aq})+2 \mathrm{KOH}(\mathrm{aq}) \rightarrow \mathrm{K}_{2} \mathrm{SO}_{4}(\mathrm{aq})+\mathrm{Cu}(\mathrm{OH})_{2}(\mathrm{~s})$

The copper sulfate reactant mixture was prepared by dissolving $1.0 \mathrm{~g}$ of Pluronic F68 into $10 \mathrm{~mL}$ of 0.5 $\mathrm{M} \mathrm{Cu}(\mathrm{II}) \mathrm{SO}_{4}$ aqueous solution [Sigma-Aldrich, www.sigma-aldrich.com] and was loaded into a microcontainer. The potassium hydroxide reactant mixture was prepared by dissolving $1.0 \mathrm{~g}$ of Pluronic F68 into $10 \mathrm{~mL}$ of $1.0 \mathrm{M} \mathrm{KOH(aq)} \mathrm{and} \mathrm{was} \mathrm{loaded} \mathrm{into} \mathrm{a} \mathrm{second} \mathrm{microcontainer.} \mathrm{The} \mathrm{microcontainers}$ were placed in close proximity into a poly (dimethyl siloxane) [PDMS, Dow Corning Sylgard® ${ }^{\circledR}$ 184, www.dowcorning.com] microwell. The microwell was fabricated by molding PDMS against an SU-8 photoresist master. The diffusion and reaction medium was water.

Phenolphthalein-KOH Reaction (Fig. 3d-f): The indicator mixture for the phenolphthalein-KOH reaction was prepared by adding $0.25 \mathrm{~mL}$ of phenolphthalein solution to an aqueous polymeric solution composed of $1.0 \mathrm{~g}$ of Pluronic F68 dissolved in $10 \mathrm{~mL}$ water. The alkaline mixture was prepared by adding $0.5 \mathrm{~mL}$ of $4 \mathrm{M} \mathrm{KOH(aq)} \mathrm{[Sigma-Aldrich,} \mathrm{www.sigma-aldrich.com]} \mathrm{to} \mathrm{an} \mathrm{aqueous} \mathrm{polymeric} \mathrm{solution} \mathrm{composed}$ of 1.0 g Pluronic F68 and $10 \mathrm{~mL}$ water. Two containers were loaded with the phenolphthalein solution and one with the $\mathrm{KOH}$ solution. The three containers were then placed into a PDMS microwell, with water as the diffusion and reaction medium. The reactions were also imaged using a stereozoom binocular microscope. 